\title{
ANALYSIS OF COHERENT OPTICAL TRANSITION RADIATION INTERFERENCE PATTERNS PRODUCED BY SASE-INDUCED MICROBUNCHES
}

\author{
D.W. Rule ${ }^{\dagger}$, Naval Surface Warfare Center, Carderock Division, West Bethesda, MD 20817-5700 \\ A.H. Lumpkin*, Advanced Photon Source, ANL, Argonne, IL 60439 USA
}

\begin{abstract}
We present an analysis of data published recently on coherent optical transition radiation interferometry (COTRI) angular distributions made on the Argonne experiment on microbunching [1]. These COTRI distributions exhibited some intriguing characteristics: highly enhanced lobe patterns in the $+/-\theta_{y}$ direction, instead of circularly symmetric fringes; and asymmetries in intensities in $+\theta_{y}$ vs. $-\theta_{y}$ in some cases. We have done calculations that produce patterns consistent with those observed. The role of the microbunch's elliptical transverse beam profile in accounting for the $\theta_{x}-\theta_{y}$ asymmetry is illustrated. Similarly, a beam profile with a $+/-\mathrm{y}$ asymmetry gives a lobe asymmetry in $+\theta_{y}$ vs. $-\theta_{y}$. The interplay of the interference fringe pattern and the microbunch's transverse form factor accounts for the angular positions of the lobes. The fringe visibility was calculated by convolving the beam divergence distribution with the COTRI pattern. Thus, we have shown that COTRI angular patterns can be used to characterize the effective transverse size, microbunch length, divergence, and the bunching fraction.
\end{abstract}

\section{INTRODUCTION}

In a previously reported experiment [1], the first direct observation of the evolution of the electron-beam bunching fraction along an undulator in a self-amplified spontaneous emission free-electron laser (SASE FEL) was made using coherent optical transition radiation interferometry (COTRI). The observed SASE undulator radiation and the COTRI both exhibited exponential growth as the bunched fraction of the beam increased along the length of the undulator. Both the SASE and COTRI were observed at $537 \mathrm{~nm}$, the fundamental SASE wavelength. The COTRI fringe patterns were the first of their kind to be observed. The angular distribution of the interference fringes contained some unusual features such as highly peaked lobes in the $\theta_{y}$-direction and greatly reduced intensity along the $\theta_{x}$-direction (see Fig. 3 of [1]). Another intriguing pattern seen under certain beam conditions was a marked asymmetry in the intensity of the lobes in the $+\theta_{y}$ vs. $-\theta_{y}$ direction. Here we present

†ruledw@nswccd.navy.mil

*Work supported by the U.S. Department of Energy, Office of Basic Energy Sciences, Contract No. W-31-109-ENG-38. calculations of COTRI for the beam parameters of the electron-beam microbunching experiment in Ref. 1, which show the same behavior as was observed. Our results indicate that beam diagnostics based on optical transition radiation interferometry (OTRI) can be extended to the coherent radiation production regime and can include transverse microbunch profile measurements in the context of SASE-FEL experiments. This application is in addition to the previously demonstrated uses of OTR for electron beam emittance [2], bunch length, and bunching fraction measurements [3].

\section{COTRI CALCULATIONS}

We will present calculations of coherent optical transition radiation produced by a model macropulse with total charge $Q$ and consisting of a train of $M+1$ micropulses. Each micropulse contains $N$ electrons, and a fraction $f_{B}=N_{B} / N$ of the micropulse's electrons are bunched to form a microbunch. The expression for the spectral-angular distribution of the number of photons per unit frequency and solid angle is given by [4]:

$$
\frac{d^{2} N}{d \omega d \Omega}=\left|r_{\perp, / /}\right|^{2} \frac{d^{2} N_{1}}{d \omega d \Omega} I(\mathbf{k}) \mathfrak{I}(\mathbf{k}),
$$

where

$$
\frac{d^{2} N_{1}}{d \omega d \Omega}=\frac{e^{2}}{\hbar c} \frac{1}{\pi^{2} \omega} \frac{\left(\theta_{x}^{2}+\theta_{y}^{2}\right)}{\left(\gamma^{-2}+\theta_{x}^{2}+\theta_{y}^{2}\right)^{2}}
$$

is the single particle optical transition radiation spectralangular distribution in the case of a single interface. The reflectivity of the second interface for perpendicular and parallel polarized light is $\left|r_{\perp, / /}\right|^{2}$. The term

$$
I(\mathbf{k})=4 \sin ^{2}\left[\frac{k L}{4}\left(\gamma^{-2}+\theta_{x}{ }^{2}+\theta_{y}^{2}\right)\right]
$$

describes the interference of OTR produced at the two interfaces of a Wartski interferometer [5], separated by a distance $L$. 
The coherence function is given by

$$
\mathfrak{I}(\mathbf{k})=N+N_{B}\left(N_{B}-1\right)|H(\mathbf{k})|^{2},
$$

where

$$
H(\mathbf{k})=\frac{\rho(\mathbf{k})}{Q}=g_{x}\left(k_{x}\right) g_{y}\left(k_{y}\right) F_{z}\left(k_{z}\right) .
$$

Here, $H(\mathbf{K})$ is related to the Fourier transform of the total charge density $\rho(\mathbf{K})$, which in turn can be expressed in terms of the Fourier transforms of the $\mathrm{x}-, \mathrm{y}-$, and $\mathrm{z}-$ dependent charge form factors.

The transverse form factors are of the form

$$
g_{i}\left(k_{i}\right)=\frac{1}{\sqrt{2 \pi}} e^{-\sigma_{i}^{2} k_{i}^{2} / 2}, i=x, y,
$$

where $\sigma_{x}$ and $\sigma_{y}$ are the $\mathrm{rms} \mathrm{x}$ - and $\mathrm{y}$-dimensions transverse to the beam.

The longitudinal function is the transform of a pulse train of $M+1$ micropulses, each separated by a distance $\ell$. For our model macropulse, the transform function is

$$
F\left(k_{z}\right)=f\left(k_{z}\right) \sum_{m=0}^{M} e^{-i k_{z} m \ell}=f\left(k_{z}\right) \frac{\sin \left(M k_{z} \ell / 2\right)}{\sin \left(k_{z} \ell / 2\right)},
$$

where the microbunch longitudinal form factor is given by

$$
f\left(k_{z}\right)=\frac{1}{\sqrt{2 \pi}} e^{-\sigma_{z}^{2} k_{z}^{2} / 2},
$$

with $\sigma_{z}$ being the rms microbunch z-dimension.

The angle $\theta$ is measured between the mean electron beam axis and the direction of the wave vector $\boldsymbol{k}$. The projected angles $\theta_{x}$ and $\theta_{y}$ are in the horizontal and vertical planes, respectively. They are defined by the following expressions:

$$
\begin{aligned}
& k_{x}=k \sin \theta \cos \phi=k \sin \theta_{x} \approx k \theta_{x} \\
& k_{y}=k \sin \theta \sin \phi=k \sin \theta_{y} \approx k \theta_{y}, \\
& k_{z}=k \cos \theta \approx k=\omega / c
\end{aligned}
$$

where the last approximation is valid if $\gamma>>1$, which implies that

$$
\theta^{2}=\theta_{x}^{2}+\theta_{y}^{2}<<1
$$

Eq. 1 is numerically convolved with a Gaussian distribution of beam divergence angles in either the $x-z$ or $y-z$ plane. This affects the fringe visibility of the interference patterns and can be used to determine the beam divergence $[2,5]$. For purposes of illustration, an rms beam divergence of $0.2 \mathrm{mrad}$ was used in all the cases here. This was estimated to be close to the actual value. The wavelength was kept at $537 \mathrm{~nm}$ for all cases. The spacing between the two foils was $L=6.3 \mathrm{~cm}$ and the beam energy was $220 \mathrm{MeV}$. A bunching fraction $f_{B}=0.2 \%$ was assumed. The number of micropulses was taken to be 24 , and the micropulse charge was $60 \mathrm{pC}$.

\section{RESULTS}

Fig. 1 illustrates the effect of varying $\sigma_{y}$, the y-rms beam dimension from $51 \mu \mathrm{m}$ to $85 \mu \mathrm{m}$. As the beam becomes wider, there is a reduction in the coherent radiation appearing in the first peak, since the transform of the beam transverse spatial distribution becomes narrower in angle (see Eq. 6). Plotted for comparison is the incoherent case. Fig. 1 also shows that a beam having different transverse sizes in the $\mathrm{x}$ - and $\mathrm{y}$ - dimensions would have asymmetric radiation patterns as functions of $\theta_{x}$ and $\theta_{y}$, as was observed in [1].

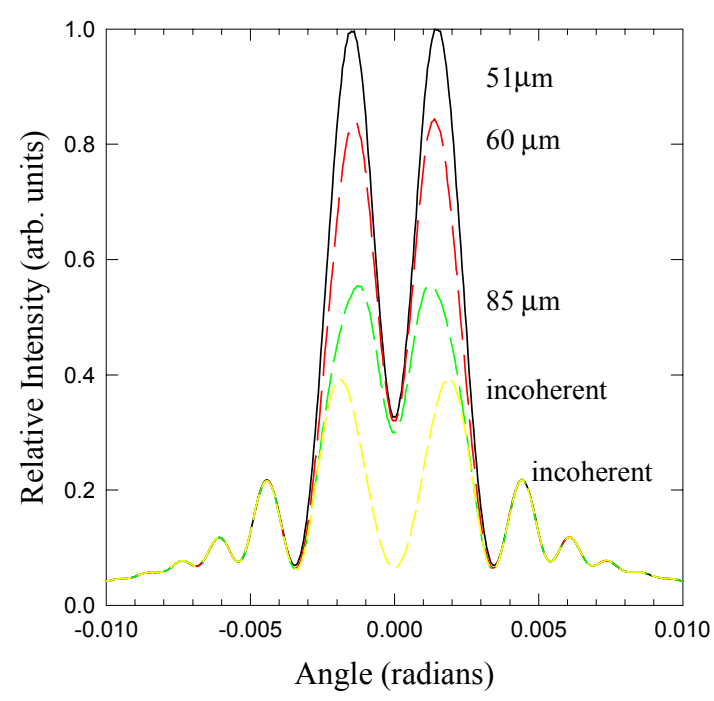

Figure 1: Effect of rms microbunch size $\sigma_{y}$ on coherent OTR interference angular pattern vs. the incoherent case.

The curve in Fig. 2 was calculated using a split Gaussian in which the value of $\sigma_{y}$ in Eq. 6 is different for negative and positive angles. The coherence factor per electron for a microbunch is plotted in Fig. 3 to illustrate the effect of an asymmetric bunch shape in the y-spatial dimension, for example. A narrower transverse ydimension results in a broader angular distribution for the Fourier transformed form factor. Again, Fig. 2 is qualitatively like data plotted in Fig. 3 of Ref. 1, for a scan along $\theta_{y}$. In Ref. 6, the higher lobe was shown to be 


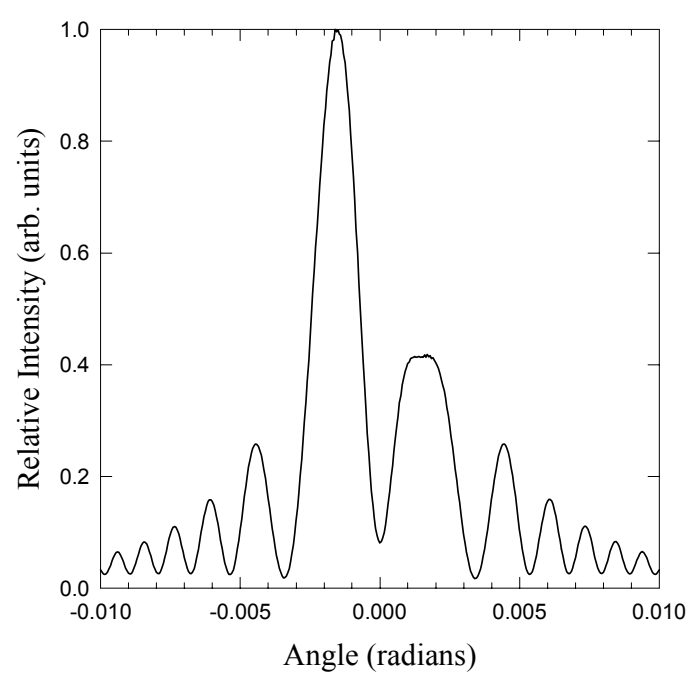

Figure 2: Effect on the interference pattern of an asymmetric transverse spatial distribution.

moveable from the $-\theta_{y}$ to the $+\theta_{y}$ side of the interference pattern by steering the electron beam slightly in the undulator.

\section{DISCUSSION}

The results presented above give a qualitative explanation of the COTRI angular patterns observed in [1] and [6] in terms of the electron beam microbunch transverse profile. The spectrum produced by coherent OTR from a single foil has been used previously to infer the longitudinal bunching in a SASE FEL experiment [3] performed with a $17 \mathrm{MeV}$ beam producing $13-\mu \mathrm{m}$ coherent radiation. Millimeter wave, coherent transition radiation was also used to determine the longitudinal and transverse dimension of a single 28ps-long micropulse, with a transverse diameter of 22-mm [7]. The coherent radiation in this case was in the 1 to $4-\mathrm{mm}$ range. Both spectral and angular data were obtained; however the angular resolution was only $52 \mathrm{mrad}$. Although two foils were used in this experiment, the foil separation was much less than the OTR coherence length, i.e. $L<<\gamma^{2} \lambda$, with $L$ as in Eq. 3, and interference fringes were not observed. Instead, two coherent peaks were observed for the 2 to $4-\mathrm{mm}$ radiation, and a single peak centered on zero angle was found in the case of 1-mm radiation.

In principle, both the coherent and incoherent OTR interference fringes can be observed. The latter provides a base line for the determination of the extent of the coherent OTR due to microbunching, and may be used to

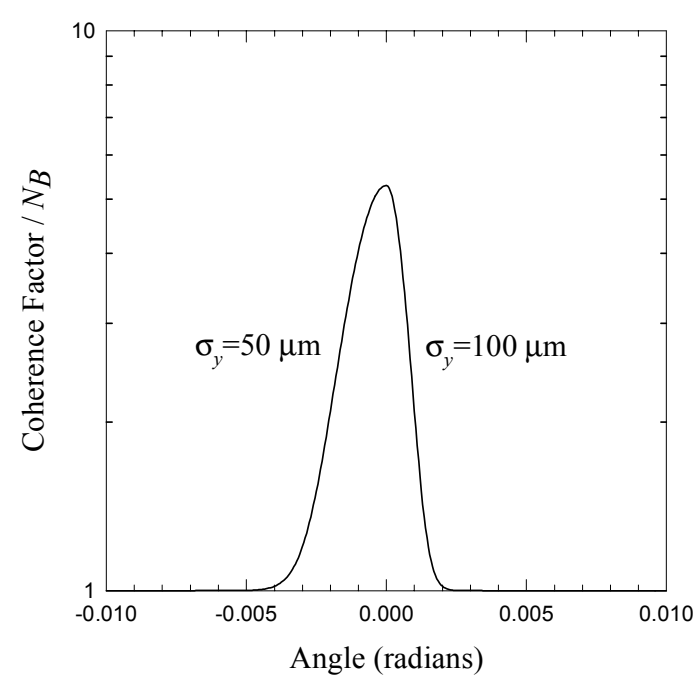

Figure 3: Asymmetric coherence factor resulting from a split Gaussian spatial profile along y-axis. See Eqs. 4-6.

infer the bunching fraction. In the case of a narrower beam radius, the coherence function can overlap more fringes producing COTRI at larger angles. The fringe spacing may be controlled at a fixed wavelength by adjusting the foil separation $L$ in Eq. 3.

Asymmetry in the lobes in the $\theta_{y}$-direction, as in Fig. 2, was controllable by slight steering of the electron beam in experiments reported in Ref. 6 . This may be due to a quadrupole focussing effect in the vertical direction in the undulator, which distorts the symmetry of the microbunches in the y-dimension. The $\mathrm{x}$-dimension of the microbunches is typically about twice as large as the y-dimension.

In summary, this analytical model for COTRI by microbuches reproduces the most significant features which have been observed in the experiments reported in Refs. 1 and 6.

\section{REFERENCES}

[1] A. H. Lumpkin, et al., PRL 86, 79-82 (2001).

[2] R. B. Fiorito and D. W. Rule, p.21 Conference Proceedings No. 319: Beam Instrumentation Workshop, Robert E. Shafer, ed. AIP (1994).

[3] A. Tremaine, et al., Phys. Rev. Lett. 81, 5816 (1998).

[4] D. W. Rule, et al., p. 745, Advanced Accelerator Concepts: Eighth Workshop, W. Lawson, C. Bellamy, and D. Brosius, eds. AIP (1999).

[5] L. Wartski, et al., J. Appl. Phys. 46, 3644 (1975).

[6] A. H. Lumpkin, et al., in these proceedings.

[7] Yukio Shibata, et al., Phys. Rev. E 50, 1479 (1994). 